\title{
Progressive Gradient Histogram Preservation Image Denoising
}

\author{
Manish Kumar Prajapati \\ Technocrats Institute of \\ Technology Bhopal, India
}

\author{
Deepak Gyanchandani \\ Technocrats Institute of \\ Technology Bhopal, India
}

\begin{abstract}
Image denoising has great significance in pre-processing step of imaging applications. Although state-of-the-art denoising methods are numerically notable and approach theoretical limits, they suffer from visible artifacts. The image denoising methods are transformed in both spatial and transformed frequency domain. Each domain has its advantages and shortcomings, which can be complemented by each other. We propose the Progressive gradient Histogram Preservation Image Denoising (PGHP) that combine both domains. This is a simple physical process, which progressively reduces noise by texture enhanced image denoising method of enforcing the gradient histogram preservation. The results with approx $1.08 \%$ improved are pointed out from the simulation.
\end{abstract}

\section{Keywords}

Image denoising, bilateral filtering, wavelet shrinkage, shorttime Fourier transform, Image denoising.

\section{INTRODUCTION}

There are many problems in image processing one of them is noise that to be denoised for the enhancement of image quality. Recently, Knaus and Zwicker established with dualdomain image denoising (DDID) methods that simple algorithms can achieve high-quality results [1]. We extend their work and propose progressive gradient histogram preservation (PGHP) for image denoising, a method motivated by recent works. BM3D and Shape-Adaptive BM3D (SA-BM3D)[2], [3].

The DDID [1] method produces high-quality results, canceled of artifacts typical to patch based methods. This method gives satisfactory results for various category images [1]. The robust estimators and wavelet shrinkage offers to explore alternative implementations of the image denoising process. In GHP image denoising, which aims to estimate the latent clean image A from its noisy observation B, is a standard yet still lively topic in image processing and low level vision. One widely used data observation model [4], [5], [6] is B = A+ $\mathrm{v}$, where $\mathrm{v}$ is additive white Gaussian noise (AWGN).One popular approach to image denoising is the VM (Variational Method), where an energy functional is minimized to explore the desired estimation of A from its noisy observation B.Motivated by the fact that natural image gradients and wavelet transform coefficients have a heavy-tailed distribution, sparsity priors are widely used in image denoising [7].The well-known total disparity minimization methods actually assume Laplacian distribution of image gradients [4].The sparse Laplacian distribution is also used to replica the high-pass filter responses and wavelet/curve let transform coefficients [8].

The proposed method combines the two domains spatial and frequency domain denoising methods. The rest of the paper is as follows. In second section the proposed work is discussed with some basics of image. Results analysis discussions are then presented in section 3 , and section 4 conclusions our exposition.

\section{PROPOSED WORK}

The denoising of image with simple model as we can take the signal $\mathrm{x}$ with white Gaussian noise $\eta$ of additive nature.

$\mathrm{Y}=\mathrm{x}+\eta$.

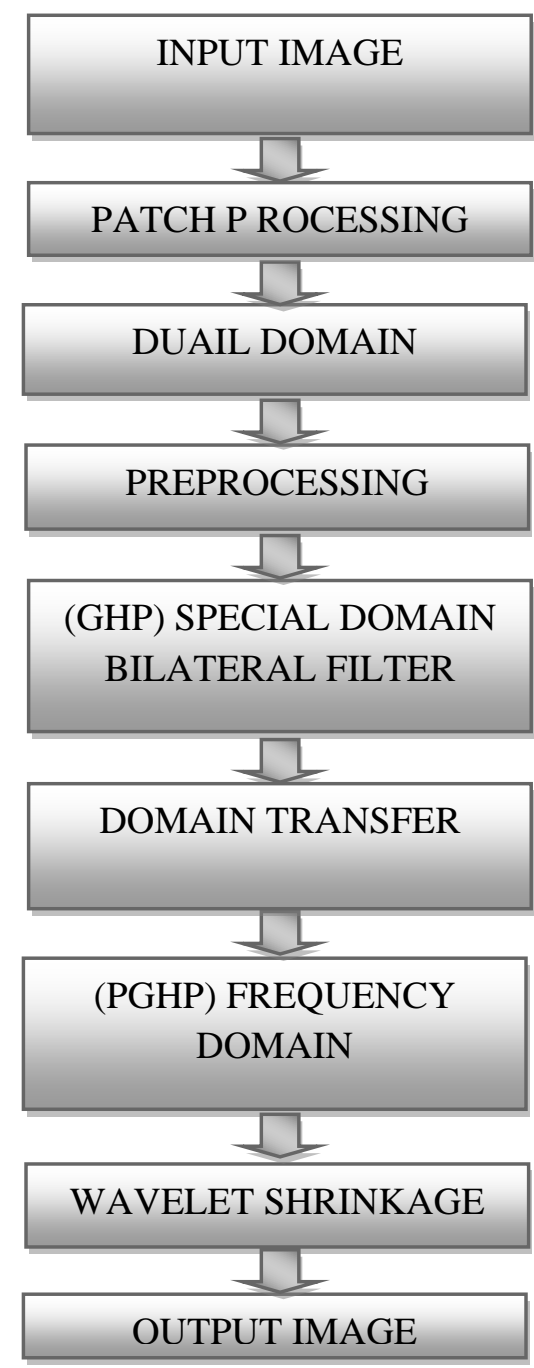

Fig.2. Block Diagram of proposed work

For the estimation process the signal $\mathrm{Y}$ can be fragmented and formulated as entropy problems. And attempted to denoise as a gradient descent with

$$
\mathrm{Y}_{\mathrm{i}+1}=\mathrm{Y}_{\mathrm{i}}-v \nabla \mathrm{E}\left(\mathrm{Y}_{\mathrm{i}}\right) \text {, }
$$

Where $v$ is the entropy adjustment -parameter. Let $A_{i}=R_{i} A$ be a patch extracted at positioni , $\mathrm{i}=1,2, \ldots, \mathrm{N}$, where $\mathrm{R}_{\mathrm{i}}$ the patch extraction operator and $\mathrm{N}$ are is the number of pixels in 
the image. Given a dictionary D, we sparsely encode the patch $A_{i}$ over $D$, resulting in a sparse coding vector $\alpha_{i}$. Once the coding vectors of all image patches are obtained, the whole image A can be reconstructed by [5]: Good priors of natural images are important to the success of an image denoising algorithm. A proper integration of different priors could further improve the denoising performance.

Here we combine both PID [9] and GHP [10] in two different domains to get the best PSNR value. In the first step take GHP in spatial domain and after that PGHP in Fourier domain. Unlike most other image denoising methods, our algorithm is simple enough that we can provide a MATLAB implementation for grayscale images in this paper. The formulation of the original DDID uses a wavelet shrinkage kernel in the frequency domain.

The result is simulated with organic image and cameraman image and noise with variance of value 40 . The entropy adjustment parameter $v$ has the influence on the PSNR. For large values of $v$ the noise is not manageable. The all other parameters are robust against change and are optimal with tolerance in the range of $0.1 \mathrm{~dB}$.

\section{RESULT ANALYSIS AND DISCUSSION}

The denoising process is explained with the starting process from a noisy image. The organic image and cameraman images are considered for this result explanation.

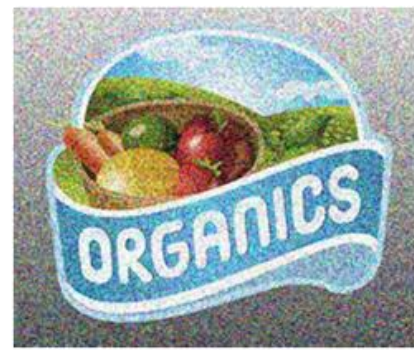

(a)

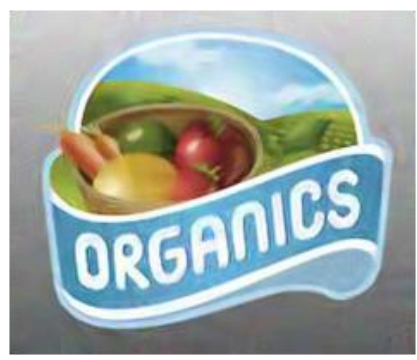

(c)

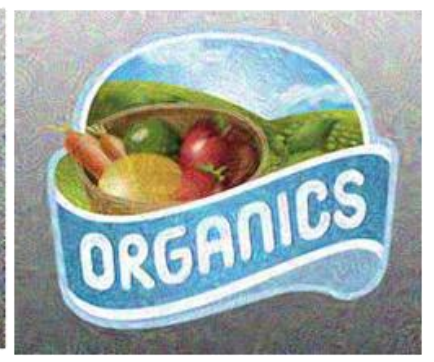

(b)

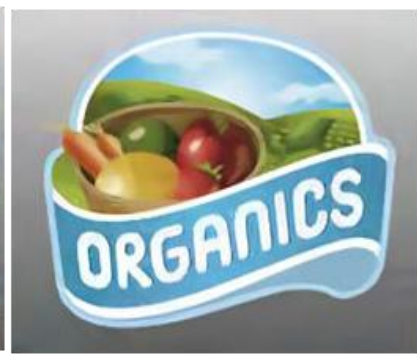

(d)
Fig.3. Progressive GHP denoising of a synthetic image by reducing the temperature $T$, given in units of the original noise variance $\sigma 2$. The noise sigma is $\sigma=40$.

First image as organic noisy image with PSNR value 16.10dB is taken. Two intermediate results with PSNR values $22.67 \mathrm{~dB}$ and $28.77 \mathrm{~dB}$ and final result with PSNR value $30.49 \mathrm{~dB}$. These results are illustrated in fig 3 .

The graphical illustration of PSNR values is given through graph 1 . This shows that proposed method gives better result for high noise density.
Table 1 Comparison of different method containing PSNR value for cameraman image

\begin{tabular}{|c|l|l|l|l|}
\hline \multirow{2}{*}{ S.NO } & \multirow{2}{*}{ METHOD } & \multicolumn{3}{|c|}{ PSNR for various $\sigma$} \\
\cline { 3 - 5 } & & $\sigma=10$ & $\sigma=25$ & $\sigma=40$ \\
\hline 1 & BM3D & 34.18 & 29.45 & 27.18 \\
\hline 2 & PGHP & 34.19 & 29.56 & 27.66 \\
\hline
\end{tabular}

The original camera man image shown in top left in fig. 4(a). The right top image fig. 4 (b) is noisy image with variance of value 25 . The image fig. 4 (d) shows the image with denoised outcome of proposed merhod.

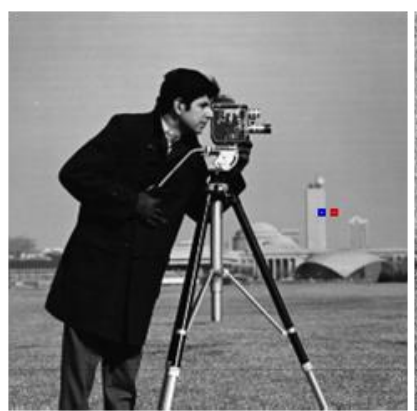

(a)

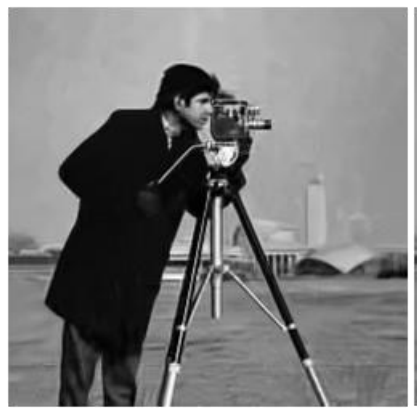

(c)

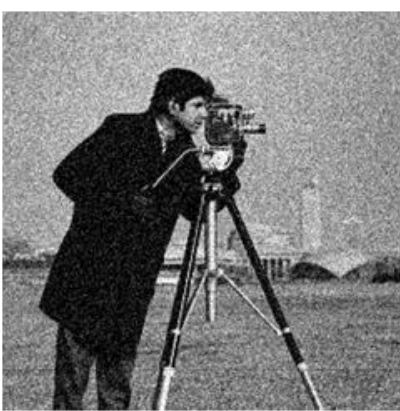

(b)

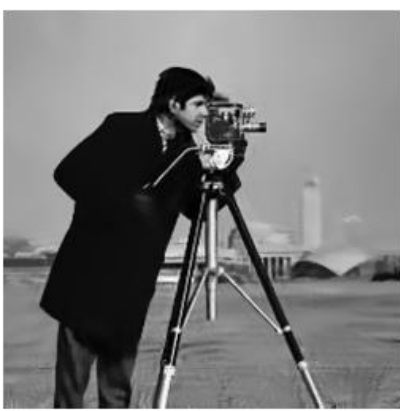

(d)
Fig. 4: Comparison of denoised Cameraman image with noise sigma $\sigma=40$.

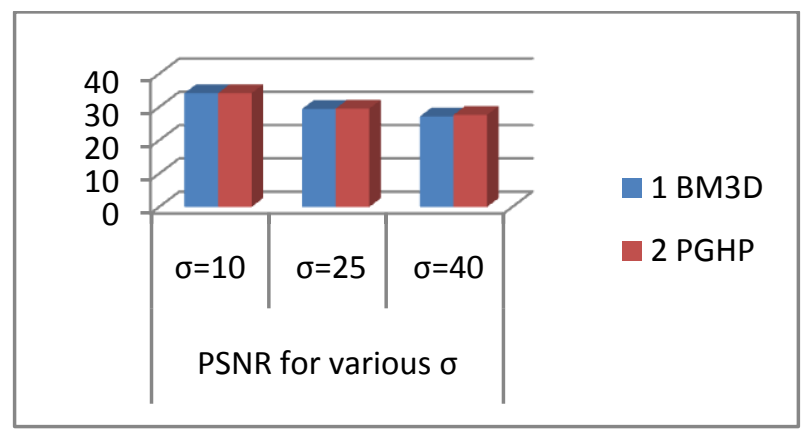

Graph: 1 Graph for Accuracy in Cameraman Image Denoising.

\section{CONCLUSION}

This paper shows a denoising of image in two domains firstly the denoising of image in spatial domain. And then the denoising is performed in frequency domain. We focused on quality and simplicity rather than performance. The image 
quality is comparatively good enough. Further work can be done for time complexity reduction.

\section{REFERENCES}

[1] C. Knaus and M. Zwicker, "Dual-domain image denoising," in Proc. 20th IEEE ICIP, Sep. 2013, pp. 440444.

[2] K. Dabov, A. Foi, V. Katkovnik, and K. Egiazarian, "Image denoising by sparse 3-d transform-domain collaborative filtering," IEEE Trans. on Image Process., vol. 16, no. 8, pp. 2080 -2095, Aug. 2007.

[3] K. Dabov, R. Foi, V. Katkovnik, and K. Egiazarian, "BM3D Image Denoising with Shape-Adaptive Principal Component Analysis," in Proc. Workshop on Signal Processing with Adaptive Sparse Structured Representations (SPARS'09), 2009.

[4] L. Rudin, S. Osher, and E. Fatemi, "Nonlinear total variation based noise removal algorithms," Phys. D, vol. 60, nos. 1-4, pp. 259-268, Nov. 1992.

[5] M. Elad and M. Aharon, "Image denoising via sparse and redundant representations over learned dictionaries," IEEE Trans. Image Process., vol. 15, no. 12, pp. 37363745, Dec. 2006

[6] V. Katkovnik, A. Foi, K. Egiazarian, and J. Astola, "From local kernel to nonlocal multiple-model image denoising," Int. J. Computer. Vision, vol. 86, no. 1, pp. 1-32, Jan. 2010
[7] R. Fergus, B. Singh, A. Hertzmann, S. Roweis, and W. T. Freeman, "Removing camera shake from a single photograph," in Proc. ACMSIGGRAPH, 2006, pp. 787794.

[8] M. Wainwright and S. Simoncelli, "Scale mixtures of Gaussians and the statistics of natural images," in Proc. NIPS, vol. 12. 1999, pp. 855-861.

[9] C. knaus, , M. Zwicker "Progressive Image Denoising", IEEE Trans. on Image Processing, vol. 23, no.7, Jul. 2014.

[10] W. Zeo, "Gradient Histrogram Estimation And Preservation For Texture Enhanced Image Denoising", IEEE Trans. on Image Process., vol. 23, no.6, Jun. 2014.

\section{AUTHOR PROFILE}

Manish Kumar Prajapati received the B.E. degree in Electronics \& communication engineering from NRI Institute information Science \& Technology Bhopal India in 2012.He is currently pursuing the $\mathrm{M}$. Tech degree from Technocrats Institute of Technology Bhopal, India. His current research interests image restoration.

Deepak Gyanchandani completed B.E. degree in Electronics and Communication from LNCTS, Bhopal in 2010 \& M.E. degree in Electronics and Communication from SGSITS Indore. He is currently working as assistant professor in Technocrats Institute of Technology Bhopal, India. 